\title{
New Strategies in Controlling Drug Resistance
}

\author{
David Frame, PharmD
}

\begin{abstract}
BACKGROUND: Chronic myeloid leukemia (CML) is most often caused by the translocation of chromosomes 9 and 22 to create the fusion protein, BCR-ABL. This constitutively active tyrosine kinase promotes cell division and blocks apoptosis, leading to unregulated growth of hematopoietic stem cells. Imatinib is a small molecule that binds to BCR-ABL at the site in which adenosine triphosphate (ATP) binds and blocks BCR-ABL function by blocking its ability to use ATP. As a front-line therapy, imatinib has been tremendously successful, with $80 \%$ to $90 \%$ of patients with chronic phase (CP) CML remaining progression free for more than 5 years. Increasingly, however, imatinib-resistant clones are appearing that allow the disease to progress. Dealing with the rise of these resistant clones has presented an important challenge to health care providers.
\end{abstract}

OBJECTIVE: To review the mechanisms by which CML becomes resistant to imatinib and to discuss the new therapeutic alternatives to imatinib and when they should be considered.

SUMMARY: Managed care weighs advances and associated costs to determinThe introduction of imatinib has indefinitely lengthened the survival time of patients with $\mathrm{CML}$, transforming this into a chronic disease condition. However, care must be taken to avoid the appearance of imatinib-resistant clones. Resistance can manifest through 1 of several mechanisms, including increased plasma protein binding, increased drug efflux, the appearance of BCR-ABL mutants that have low affinity for imatinib, the appearance of BCR-ABL independent proliferation signals, and the amplification of the BCR-ABL gene. Subtherapeutic dosing is highly likely to result in the selection of a resistant clone; thus, it is of paramount importance to ensure the imatinib dose is sufficient. Measurements of plasma levels of imatinib are proving to be predictive of outcomes, suggesting that the monitoring of imatinib levels will be an important and necessary aspect of monitoring disease. Several clinical trials have shown that high-dose imatinib provides greater and faster response rates. This also may lead to better long-term blockade of disease progression. Waiting until disease progression begins appears to lead to greater resistance to high-dose imatinib and should be avoided. Dasatinib is a next-generation kinase inhibitor that binds to both SRC and to multiple conformations of BCR-ABL. It is capable of blocking several BCR-ABL mutants that are resistant to imatinib. Clinical trials have shown dasatinib is effective in maintaining patients in $\mathrm{CP}$ and can return a percentage of patients with advanced CML to CP. Economic analysis indicates that the cost-efficacy ratio for imatinib is approximately $\$ 40,000$ per year and compares favorably with the costs of accepted procedures, such as dialysis. Data have shown that tyrosine kinases also have better mortality rates than allogeneic bone marrow transplant for the first 8 years and appear to also be more cost-effective than transplantation for this time frame.

CONCLUSION: New clinical data are beginning to supply us with effective dosing and monitoring parameters for imatinib and dasatinib treatment of $\mathrm{CML}$. Economic analysis indicates that these therapies are acceptable in cost and effective in providing good quality of life to patients.

KEYWORDS: Dasatinib, Drug cost, Drug resistance, Imatinib, Leukemia

J Manag Care Pharm. 2007;13(8)(Suppl S-a):S13-S17

Copyright@ 2007, Academy of Managed Care Pharmacy. All rights reserved.

Author

DAVID FRAME, PharmD, is a clinical hematology, oncology, and blood and marrow transplant specialist, and an assistant professor of pharmacy, University of Michigan Health System, Ann Arbor, MI. hronic myeloid leukemia (CML) has now truly become a chronic disease. Before the availability of imatinib, the average survival of the newly diagnosed patient was 3 to 6 years. Now, 80\% to $90 \%$ of patients are remaining in chronic phase (CP) after 5 years. This creates important issues for the managed care pharmacist, including how these patients will be monitored, how a decision will be made to change therapy, and what costs will be associated with this new population of patients with chronic disease. A change in therapy could be motivated by toxicity to the front-line drug, but most often a change is made because of the appearance of resistant clones. This review will focus first on the emergence of imatinib resistance and newer therapies in clinical trials that can, to some extent, block the activity of these mutated BCR-ABL proteins. Second, the economics of these newer therapies will be compared.

\section{Mechanisms of Imatinib Resistance}

There are 5 main mechanisms currently known that may result in imatinib resistance. The first is plasma protein binding. Imatinib binds very strongly to the $\partial$-1-acid glycoprotein. ${ }^{1}$ Changes in $\partial$-1-acid glycoprotein may change the amount of binding of this drug, thus changing drug availability. The second mechanism is drug efflux. ${ }^{2}$ Imatinib is a substrate for the P-glycoprotein pump, which can result in decreased intracellular concentrations of imatinib. The third mechanism is the mutation of the BCR-ABL kinase. ${ }^{3}$ Mutations are common in dividing cancer cells. In the presence of imatinib, cells that generate mutations in BCR-ABL can overcome the ability of this drug to inhibit cell division. Mutations that alter the imatinib binding site without affecting the adenosine triphosphate binding site or the active site of the kinase are very effective at inducing drug resistance. The fourth mechanism is independent of BCR-ABL. Although the 9:22 translocation is necessary to initiate CML, BCR-ABL is only one of several kinases capable of maintaining the proliferation rate of the cell while inhibiting apoptosis. Activity of other kinases and second messengers, including the SRC family of tyrosine inases, have been implicated in this form of resistance. ${ }^{4}$ The fifth mechanism is gene amplification. ${ }^{3}$ As the number of Philadelphia chromosomes increases, the number of BCR-ABL proteins expressed in the cell increases and the efficacy of imatinib decreases.

\section{Should Imatinib Levels Be Monitored?}

Subtherapeutic imatinib dosing is troubling because, similar to bacterial resistance to an antibiotic, resistant CML mutations may be selected and proliferate. Monitoring imatinib levels will be useful; however, because only a few laboratories in the United States are capable of performing these measurements, the process of monitoring will be expensive. Nevertheless, data are just now being generated, indicating that imatinib levels are quite predictive of response.

In 1 study, 68 patients with CML were examined. ${ }^{5}$ Of these, 
Response Rates in Patients With Newly Diagnosed CP CML

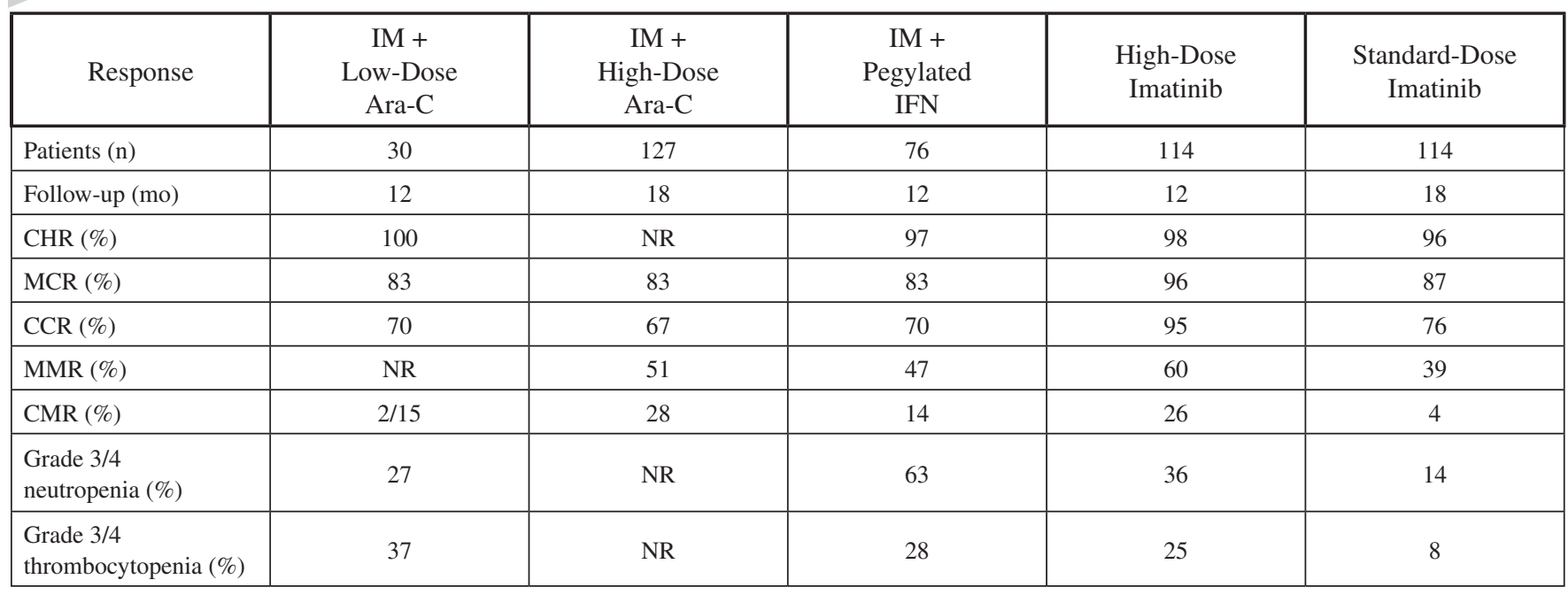

Ara-C = cytarabine $; C C R=$ complete cytogenetic remission; $C H R=$ complete hematologic response CMR = complete molecular response; $I F N=$ interferon; $I M=$ imatinib $; M C R=$ major cytogenetic response; $M M R=$ major molecular response; $N R=$ not recorded. Reprinted with permission from Deininger. ${ }^{7}$

56 were in complete cytogenetic remission (CCR) and of these, 34 had a major molecular response (MMR). Trough levels of imatinib were established for each patient, and the correlation between imatinib trough and degree of response was found to be highly significant. More recently, data from the International Randomized Interferon versus STI571 (IRIS) study demonstrated that the trough concentration (Cmin) of imatinib was significantly higher in patients who had achieved a CCR (1,009 ng/mL vs. $812 \mathrm{ng} / \mathrm{mL}, \mathrm{P}=0.0116){ }^{6}$ Blood samples had been obtained from 551 patients with CML on day 1 (trough sample taken 24 hours after the first dose) and at steady state on day 29 of treatment. Molecular response rates after 1 year showed that only $25 \%$ of patients with levels $<647 \mathrm{ng} / \mathrm{mL}$ went into a molecular response, whereas $40 \%$ of patients with levels $>647 \mathrm{ng} / \mathrm{mL}$ went into a molecular response. After 4 years, 53\% of patients with a low Cmin achieved an MMR versus $80 \%$ of patients with a high Cmin. Each of these patients was given an imatinib dose of 400 mg, but their plasma levels, as well as their responses, varied considerably.

\section{Utility of High-Dose Imatinib Therapy}

One strategy that might address the issue of resistance is to increase the imatinib dose. Data supporting this hypothesis consisted of a comparison of 5 independent studies. ${ }^{7}$ The study compared the standard $400 \mathrm{mg}$ dose of imatinib with imatinib plus either cytarabine or interferon $\partial$ (IFNO) or with high-dose imatinib (600-800 mg). The response rates are shown in the Table. Adding cytotoxic chemotherapy did not improve the overall response to therapy compared with imatinib alone and greatly increased the incidence of grade 3 and 4 neutropenia and throm- bocytopenia. High-dose imatinib produced the best results of all. An MMR was achieved by $60 \%$ of patients at the higher imatinib dose compared with 39\% at the standard dose.

In a second trial (the RIGHT trial), 115 patients with CP CML received initial dosing of 800 mg imatinib. ${ }^{8}$ At 16-month median follow-up, it was found that these patients had a rapid MMR. By 6 months, 44\% had achieved an MMR compared with patients in the IRIS trial, in which only $21 \%$ of patients had achieved an MMR at 6 months on the standard imatinib dose. It should be noted that 10 patients had to discontinue therapy as a result of adverse events. Is the rapidity of the MMR worth the increased toxicity? One would predict that the answer would be yes. Each surviving leukemia cell has the capability to become resistant. With the rapid reduction in the number of cells, the probability of a resistant clone arising may be decreased. Whether or not the initial high-dose therapy will decrease time to resistance will be determined over the course of the long-term monitoring of these patients.

Patients with CML in CP can be ranked as low or high risk as measured by their Sokal score, which is calculated using age, spleen size, platelet count, and peripheral blood blast count. ${ }^{9}$ There are 3 categories of Sokal scores: (1) low risk $(<0.8)$; (2) intermediate risk (0.8-1.2); and (3) high risk (>1.2) ${ }^{10}$ Patients with a high score are most likely to progress to advanced disease. The next trial focused on this patient group. ${ }^{11}$ Again, patients (N = 87) were started on $800 \mathrm{mg}$ imatinib and disease was assessed after 6 and 12 months. For the Sokal trial patients, 90\% of the high-dose group achieved a CCR at 12 months, whereas $67 \%$ of high-risk patients in IRIS achieved a CCR. However, compliance was an issue. Although compliance was classified as good in the 
study, it was close to $50 \%$. In other words, approximately $50 \%$ of patients had to drop their imatinib dose to less than $600 \mathrm{mg}$ as a result of toxicities. Thus, a high initial dose of imatinib will bring about a quicker response; however, it appears difficult to keep patients at this dose level because of the likelihood of toxicities.

Because high-dose imatinib has greater toxicity, the physician might consider reserving the high-dose regimen for patients who fail therapy at $400 \mathrm{mg}$. However, this may not be as effective. In a small trial involving 20 patients with hematologic resistance or relapse at $400 \mathrm{mg}$ imatinib, patients were increased to $800 \mathrm{mg}$. Although $65 \%$ went on to achieve a complete or partial hematologic response, only 1 patient achieved a cytogenetic response (CR). ${ }^{12}$ Additionally, the response appeared to be of short duration. These data would suggest that by allowing the patient to progress to a more advanced phase of CML, the consequence would be promotion of clonal expansion of resistant cells.

\section{Dasatinib}

Dasatinib is structurally different from imatinib, and it has some very different properties from imatinib. It not only inhibits the BCR-ABL kinase, but also inhibits SRC kinase. ${ }^{13}$ It binds the BCR-ABL kinase domain in the active and inactive confirmation. Imatinib only binds if the kinase is in an inactive form. This becomes important when one considers how resistant mutations block imatinib function. Many of the mutations are located in sites around the kinase that shift the formation of the kinase to an active formation. Thus, these mutations not only make the kinase more active, they block the ability of imatinib to bind. Dasatinib binds to BCR-ABL with a 300-fold increase in affinity, and because it binds BCR-ABL in the active conformation, it is capable of inhibiting cells that develop many of the imatinibresistant mutations.

The phase I trial was performed on 84 patients with CML, of whom 72 (86\%) were resistant to imatinib therapy. ${ }^{13}$ The primary objective of the trial was to define the safety and tolerability of dasatinib with antileukemic activity and correlation to BCRABL mutations as a secondary objective. Dasatinib (15-240 mg) was administered orally in 4-week treatment cycles once or twice daily. Interestingly, although complete hematologic response (CHR) was achieved in 92\% of patients in CP, CHR also was achieved in $45 \%$ of patients in accelerated phase (AP) and 35\% of patients in blast phase (BP) CML. This achievement of CHR stands in contrast to imatinib trials that were much less effective in advanced CML..$^{14,15}$ Indeed, whereas virtually no patients with advanced CML achieved a CCR in imatinib trials, $18 \%$ and 26\% of patients in AP and BP, respectively, achieved a CCR in this phase I dasatinib trial.

An update for this trial was recently reported. ${ }^{16}$ After a median follow-up of 13 months, progression-free survival of all patients was $90 \%$. Ninety-one percent of patients achieved a CHR, whereas $58 \%$ achieved a major CR. Of these, $41 \%$ had never achieved a CR at all on imatinib. Dose interruptions occurred in
331 patients (86\%), and dose reductions occurred in 269 (70\%), with an average daily dose of $103 \mathrm{mg}$ per day. The major adverse event was pleural effusion, which affected $6 \%$ of patients. It is likely that these extraordinary results reflect the greater affinity of dasatinib, as well as its ability to bind to SRC, which is another major kinase signaling pathway that mediates cancer cell growth.

To answer the question of how to treat patients who were imatinib resistant at 400 to $600 \mathrm{mg}$, a study was done comparing high-dose imatinib to dasatinib in this setting. One hundred fifty patients who were resistant to low-dose imatinib were randomized and treated with dasatinib (70 mg twice daily) or imatinib (800 mg daily). ${ }^{17}$ With a minimum follow-up of 10 months, CHR rate was $92 \%$ with dasatinib versus $82 \%$ with imatinib. Major CR rate was $48 \%$ with dasatinib versus $33 \%$ with imatinib, and CCR rate was $35 \%$ with dasatinib versus $16 \%$ with imatinib. In patients with no prior CR, 44\% achieved major CR with dasatinib versus $7 \%$ with high-dose imatinib. Thus, the results clearly demonstrated that dasatinib is superior to high-dose imatinib for patients with CML who have become resistant to the lower imatinib dose. Another study has demonstrated 100\% 2-year survival in 125 imatinib-resistant patients when treated with subsequent dasatinib or nilotinib versus $72 \%$ survival with allogeneic transplant or $67 \%$ with other therapies. ${ }^{18}$

The optimal dasatinib dose is still a matter of debate. Currently, the approved dose is $70 \mathrm{mg}$ twice a day; however, almost 50\% of patients experience hematologic toxicities, such as neutropenia and thrombocytopenia and drop to $50 \mathrm{mg}$ per day. This has propelled a trial in which 4 dasatinib dosing regimens (50 mg or $70 \mathrm{mg}$ twice daily vs. $100 \mathrm{mg}$ or $140 \mathrm{mg}$ once daily) are being compared. ${ }^{19}$ The trial is ongoing and at present the interim results cannot distinguish between the various doses. The results of this trial are important as they will affect dasatinib dosing in the future.

\section{New Thoughts in CML}

\section{Bone Marrow Transplant Versus Best Drug}

Imatinib and dasatinib are tremendously effective at blocking disease progression; however, they are generally not thought to be curative. Patients live with the possibility of disease recurrence for the remainder of their lives. Currently, the only known curative therapy is allogeneic bone marrow transplant (BMT). However, this is only a potential option for younger patients in good performance status. Even in these patients, the early chance of mortality appears to be greater in those who undergo BMT. To establish relative risks for these 2 strategies, 621 newly diagnosed patients were followed. ${ }^{20}$ Thirty-eight percent had a matched related donor and chose BMT, whereas the remainder received best drug therapy. At the beginning of this trial, the best drug therapy was IFNO. However, with the availability of imatinib, most of the patients switched to imatinib. Within the first year, BMT mortality was $20 \%$ to $30 \%$. Subsequently, 
patients succumbed to adverse effects, such as graft versus host disease. Indeed, the drug treatment group showed superior survival results for the first 8 years of the trial, at which point the curves began to superimpose. However, there was a higher rate of molecular remission with transplantation.

\section{Stopping Imatinib After Cytogenetic Remission}

If BCR-ABL transcripts are detectible by polymerase chain reaction (PCR), one would argue that the patient still has CML. However, in patients who achieve a complete molecular response (CMR) and no longer have detectable BCR-ABL transcripts, one could legitimately ask whether these patients still have disease. Recently, data were reported on 15 patients who had achieved a CMR for more than 2 years. ${ }^{21}$ The median duration of PCR negativity and imatinib therapy was 32 months (24-46 months) and 45 months (32-56 months), respectively. Eight patients displayed a molecular relapse with a detectable BCR-ABL transcript appearance within the first 6 months. Surprisingly, 7 other patients had an undetectable level of BCR-ABL transcripts after a median follow-up of 20 months (9-24 months). More studies are required to determine if a subset of patients may actually receive a potential cure from the tyrosine kinase inhibitors.

\section{Economics of Treating CML}

In general, the new chemotherapy drugs are extremely expensive. Imatinib costs range from $\$ 28,000$ to $\$ 60,000$ per year, depending on dosing. Dasatinib at $140 \mathrm{mg}$ per day is approximately $\$ 52,000$ per year. If one looks at the breakdown of oral chemotherapy from costs reported from 1 health care system, imatinib accounted for 29\% of claims in 2006 (Figure). ${ }^{22}$ This was actually enough for this health care system to actually triple its per-member-per month payment plan. Thus, these drugs can be extremely overwhelming in the overall insurance scheme, especially for small insurers or self-insurers.

If one examines cost utility, current published data are obsolete because they do not take into account the tremendous efficacy of the kinase inhibitors. Nevertheless, if one makes the assumption that a patient with CP CML fails IFNO and is put on a secondary therapy and then compares hydroxyurea to imatinib as that secondary therapy, cost utility analysis shows that imatinib is found to offer considerable health benefits to patients with an incremental cost-effectiveness ratio of $\$ 75,427 . .^{23}$

Dialysis is a useful maker for assessing the cost of qualityadjusted life-years. Dialysis incurs a cost of $\$ 50,000$ to $\$ 60,000$ per year, and this has been the benchmark for most cost analysis. If one looks at imatinib versus IFNo, the incremental cost-effectiveness ratio was $\$ 44,270$ per quality-of-life year gained. ${ }^{24}$ This cost is significantly less compared with some of the other biologic anticancer agents. Cetuximab, used for colon cancer, costs $\$ 30,000$ to $\$ 40,000$ for an additional 2 months of survival.

Another study estimated imatinib-mediated survival at 15.3

\section{FIGURE Distribution of Costs for Oral Chemotherapy Drugs for an Insured Health Plan}

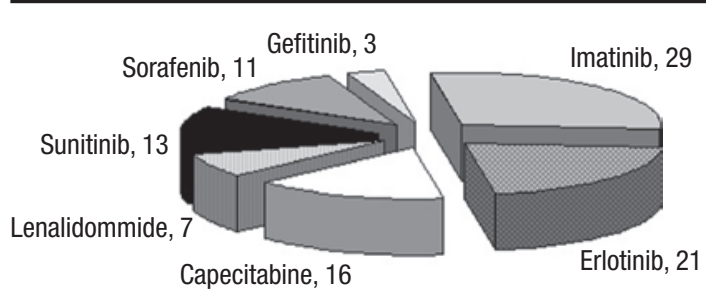

Pharmacy claims with dates of service from January 1, 2006, through May 31, 2006. Reprinted with permission from Curtiss FR. Pharmacy benefit spending on oral chemotherapy drug. J Manag Care Pharm. 2006;12(7):570-77.22

years and compared it to the survival data for IFNO of 9.1 years. ${ }^{25}$ The estimated increase in lifetime cost was calculated as approximately $\$ 241,800$. After discounting costs and survival benefits, the incremental lifetime costs were $\$ 168,100$ higher with imatinib with incremental cost-effectiveness ratios of $\$ 43,100$ per life-year saved and $\$ 43,300$ per quality-adjusted life-year saved. Finally, for BMT compared with imatinib therapy, using 2 years of data, the cost-efficiency ratio is $\$ 86,000$ for imatinib compared with $\$ 261,000$ for BMT. ${ }^{26}$

\section{Conclusions}

Imatinib has turned CML from a relatively rapidly fatal condition into a manageable chronic disease. As with all chronic conditions, disease management must be optimized. Costs must be contained by establishing appropriate dosages and identifying the important disease parameters to monitor. Recent data strongly support the monitoring of imatinib levels to establish the effective dose, as well as high-dose imatinib therapy to achieve more rapid responses and to potentially avoid the more rapid development of resistant disease. Dasatinib is a newer kinase inhibitor that binds to BCR-ABL in the active and inactive conformations, as well as to the SRC kinase, and thus can overcome the resistance caused by several common CML mutations.

Clinical trials have been quite successful, not only for patients in $\mathrm{CP}$ but to a lesser extent, for patients in advanced stages of the disease. This is most likely because of the ability of dasatinib to block SRC and BCR-ABL kinases. Economic analysis shows that imatinib and dasatinib therapies have a cost-efficacy ratio close to that of dialysis, a marker for accepted costs of quality-of-life years. The cost-efficacy ratio is far superior to the older chemotherapies, such as hydroxyurea and IFNO, and also appears to be superior to BMT. Finally, it appears possible that some patients who have achieved a CMR with imatinib can stop therapy without relapse. More time is needed before it can be determined whether these drugs indeed have a curative effect. 


\section{DISCLOSURES}

This article is based on a presentation given by the author at a symposium held during the Academy of Managed Care Pharmacy's 19th Annual Meeting and Showcase on April 12, 2007, in San Diego, CA.

The author has served as a consultant for and received honoraria from Bristol-Myers Squibb and Novartis.

\section{UNLABELED/UNAPPROVED USES OF DRUGS}

The author has cited applications and dosing of imatinib that are not approved by the U.S. Food and Drug Administration (i.e., 600-800 mg dosing for first-line therapy and $800 \mathrm{mg}$ for refractory patients).

\section{REFERENCES}

1. Gambacorti-Passerini C, Zucchetti M, Russo D, et al. Alpha-1-acid glycoprotein binds to imatinib (STI571) and substantially alters its pharmacokinetics in chronic myeloid leukemia patients. Clin Cancer Res. 2003;9:625-32.

2. Thomas J, Wang L, Clark RE, Pirmohamed M. Active transport of imatinib into and out of cells: implications for drug resistance. Blood. 2004;104:3739-45.

3. Gorre ME, Mohammed M, Ellwood K, et al. Clinical resistance to STI-571 cancer therapy caused by BCR-ABL gene mutation or amplification. Science. 2001;293:876-80

4. Cortes J, Kantarjian H. New targeted approaches in chronic myeloid leukemia. J Clin Oncol. 2005;23:6316-24

5. Picard S, Titier K, Etienne G, et al. Trough plasma imatinib concentrations are associated with both cytogenetic and molecular responses to standard-dose imatinib in chronic myeloid leukemia. Blood (ASH Annual Meeting Abstracts). 2006;108:Abstract 2141.

6. Larson RA, Druker BJ, Guilhot F, et al. Correlation of pharmacokinetic data with cytogenetic and molecular response in newly diagnosed patients with chronic myeloid leukemia in chronic phase (CML-CP) treated with imatinib-an analysis of IRIS study data. Blood (ASH Annual Meeting Abstracts). 2006;108:Abstract 429

7. Deininger MWN. Management of early stage disease. Hematology. 2005;2005:174-82

8. Cortes J, Giles F, Salvado AJ, et al. Molecular responses in newly diagnosed chronic myelocytic leukemia (CML) patients treated with $800 \mathrm{mg}$ imatinib daily: an update from the RIGHT trial study group. Blood (ASH Annual Meeting Abstracts). 2006;108:Abstract 2149.

9. Sokal JE, Baccarani M, Russo D, Tura S. Staging and prognosis in chronic myelogenous leukemia. Semin Hematol. 1988;25:49-61.

10. Besa EC, Woermann U. Chronic myelogenous leukemia. eMedicine. Available at: http://www.emedicine.com/med/topic371.htm. Accessed August 14, 2007

11. Rosti G, Castagnetti F, Amabile M, et al. Imatinib high dose (800 $\mathrm{mg}$ ): results of a phase II trial of the GIMEMA (Gruppo Italiano Malattie Ematologiche Dell'Adulto) CML working party in intermediate sokal risk patients and status-of-the-art of an ongoing multinational, prospective randomized trial of imatinib standard dose (400 mg daily) vs high dose (800 mg daily) in high sokal risk patients. Blood (ASH Annual Meeting Abstracts). 2006;108:Abstract 4776

12. Kantarjian HM, Talpaz M, O'Brien S, et al. Dose escalation of imatinib mesylate can overcome resistance to standard-dose therapy in patients with chronic myelogenous leukemia. Blood. 2003;101:473-75.
13. Talpaz M, Shah NP, Kantarjian H, et al. Dasatinib in imatinib-resistant Philadelphia chromosome-positive leukemias. N Engl J Med. 2006;354:253141 .

14. Talpaz M, Silver RT, Druker BJ, et al. Imatinib induces durable hematologic and cytogenetic responses in patients with accelerated phase chronic myeloid leukemia: results of a phase 2 study. Blood. 2002;99:1928-37.

15. Sawyers CL, Hochhaus A, Feldman E, et al. Imatinib induces hematologic and cytogenetic responses in patients with chronic myelogenous leukemia in myeloid blast crisis: results of a phase II study. Blood. 2002;99:3530-39.

16. Baccarani M, Kantarjian HM, Apperley JF, et al. Efficacy of dasatinib (SPRYCEL $®$ ) in patients (pts) with chronic phase chronic myelogenous leukemia (CP-CML) resistant to or intolerant of imatinib: updated results of the CA180013 'START-C' phase II study. Blood (ASH Annual Meeting Abstracts). 2006;108:Abstract 164

17. Shah N, Pasquini R, Rousselot P, et al. Dasatinib (SPRYCEL $®)$ vs escalated dose of imatinib (im) in patients (pts) with chronic phase chronic myeloid leukemia (CP-CML) resistant to imatinib: results of the CA180-017 START-R randomized study. Blood (ASH Annual Meeting Abstracts). 2006;108: Abstract 167.

18. Kantarjian H, O'Brien S, Talpaz M, et al. Outcome of patients with Philadelphia chromosome-positive chronic myelogenous leukemia post-imatinib mesylate failure. Cancer. 2007;109:1556-60.

19. Hochhaus A, Kim DW, Rousselot P, et al. Dasatinib (SPRYCEL $(5)$ $\mathrm{mg}$ or $70 \mathrm{mg}$ BID versus $100 \mathrm{mg}$ or $140 \mathrm{mg}$ QD in patients with chronic myeloid leukemia in chronic phase (CML-CP) resistant or intolerant to imatinib: results of the CA180-034 study. Blood (ASH Annual Meeting Abstracts). 2006;108:Abstract 166

20. Hehlmann R, Pfirrmann M, Hochhaus A, et al. Randomized comparison of primary allogeneic stem cell transplantation and best available drug treatment in chronic myeloid leukemia. Blood (ASH Annual Meeting Abstracts). 2006;108:Abstract 427.

21. Mahon F-X, Huguet F, Etienne G, et al. Imatinib mesylate discontinuation in patients with chronic myelogenous leukemia in complete molecular remission: an update follow up. Blood (ASH Annual Meeting Abstracts). 2006; 108:Abstract 2154.

22. Curtiss FR. Pharmacy benefit spending on oral chemotherapy drugs. J Manag Care Pharm. 2006;12:570-77. Available at: www.amcp.org/data/jmcp/ contemporary_subjects_570_577.pdf.

23. Warren E, Ward S, Gordois A, Scuffham P. Cost-utility analysis of imatinib mesylate for the treatment of chronic myelogenous leukemia in the chronic phase. Clin Ther. 2004;26:1924-33.

24. Dalziel K, Round A, Garside R, Stein K. Cost effectiveness of imatinib compared with interferon-alpha or hydroxycarbamide for first-line treatment of chronic myeloid leukaemia. Pharmacoeconomics. 2005;23:515-26.

25. Reed SD, Anstrom KJ, Ludmer JA, et al. Cost-effectiveness of imatinib versus interferon-alpha plus low-dose cytarabine for patients with newly diagnosed chronic-phase chronic myeloid leukemia. Cancer. 2004;101:257483

26. Skrepnek GH, Ballard EE. Cost-efficacy of imatinib versus allogeneic bone marrow transplantation with a matched unrelated donor in the treatment of chronic myelogenous leukemia: a decision-analytic approach. Pharmacotherapy. 2005;25:325-34 